\title{
Primary Cicatricial Alopecia Successfully Treated with A Combination of Oral Zinc with Nd: YAG Laser. A Case Series of Five Patients
}

\author{
Rosa Giménez-García ${ }^{1 *}$, Dra Teresa E. Lázaro Cantalejo² \\ ${ }^{1}$ Clinical Assitant, Hospital Rio Hortega. Calle Dulzaina s/n. 47012Valladolid. Spain \\ ${ }^{2}$ Dermatologist, Dermatologic Center Santa Teresa. C/ Perú n ${ }^{\circ}$ 15.47004,Valladolid. Spain
}

*Corresponding Author: Rosa Giménez-García, Clinical Assitant, Hospital Rio Hortega. Calle Dulzaina s/n.47012Valladolid. Spain, Email: rosagim@hotmail.com

\begin{abstract}
Cicatricial alopecia refers to a group of uncommon inflammatory hair loss disorders which are characterized by the destruction of the hair follicle unit that is replaced by fibrous tissue and result in permanent hair loss.

We describe five primary cicatricial alopecia (PCA) cases with an excellent response to oral zinc combined with long-pulsed Nd:YAG 1064-nm laser treatment.

A working classification of PCA, mainly based on the most representative pathological finding of scalp biopsy sample include chronic cutaneous lupus erythematosus, lichen planopilaris (LPP), classic pseudopelade of Brocq, central centrifugal cicatricial alopecia, alopecia mucinosa and keratosis follicularis spinulosa decalvans as "lymphocytic" PCA. Frontal fibrosing alopecia and Graham-Little syndrome were considered as LLP variants. The neutrophilic PCA group comprises FD and dissecting cellulitis/folliculitis. Acne keloidalis, acne necrotica and eruptive pustular dermatosis were classified as mixed cell infiltrate PCA. The evaluation is challenging and the treatment may be frustrating. The aim of treatment currently focuses the reduction of symptoms and to slow the progression of the inflammatory waves and the scarring process at the earliest phase.
\end{abstract}

In our opinion a combination of oral zinc and laser therapy may be an option for the treatment of some cases of $P C A$

Keywords: Scarring alopecia, Folliculitis decalvans, Dissecting cellulitis, Laser therapy

\section{INTRODUCTION}

Cicatricial (Scarring) alopecia (CA) refers to a group of uncommon inflammatory hair loss disorders which are characterized by the destruction of the hair follicle unit that is replaced by fibrous tissue and result in permanent hair loss. Clinically there is loss of visible follicular ostia. CAs are classified as primary cicatricial alopecia (PCA), secondary cicatricial alopecia and development/hereditary CA. In primary cicatricial alopecia (PCA), the hair follicle is the main target for destruction with sparing of the interfollicular reticular dermis. The inflammatory cells target and destroy the stem cells in the bulge area of hair follicles. PCA are divided into subgroups depending upon the predominant inflammatory infiltrates. The neutrophilic PCA group comprises folliculitis decalvans (FD) and dissecting cellulitis/folliculitis of the scalp (perifolliculitis abscedens et suffodiens) [1-5]. We here report five cases PCA showing complete remission after treatment based on oral zinc in combination with $\mathrm{Nd}$ :YAG laser

\section{CASES RePorts}

\subsection{Case 1}

A 22-year-old man, with a family history of melanoma and diabetes and personal history of vancomicyn allergy, obesity, as well as axillary hidrosadenitis, presented to us with a $3 \times 5 \mathrm{~cm}$ area of cicatricial alopecia at the scalp in the vertex. The patient complaint of suppuration for approximately 2-3 months. Bacterial culture showed a growth of citrobacter kosery. He had been treated with topical clindamycin without improvement. Histopathological study was consistent with suppurative folliculitis. We 
Primary Cicatricial Alopecia Successfully Treated with A Combination of Oral Zinc with Nd: YAG Laser. A Case Series of Five Patients

diagnosed dissecting cellulitis and the patient was treated with a 6-month course of gluconate zinc $70 \mathrm{mg}$ daily and laser therapies. A total of 10 treatments in an 2 week inter-treatment interval, with long-pulsed Nd:YAG 1064-nm laser, 3,5 and $10-\mathrm{mm}$ spot size, the energy density from $50 \mathrm{jul} / \mathrm{cm}^{2}$ to $150 \mathrm{jul} / \mathrm{cm}^{2}$, and pulse duration between 20 to $30 \mathrm{~ms}$. The therapeutic result was very good with remission of the inflammation and partial recovering of hair. (Fig 1) As maintenance therapy, he is being treated with gluconate zinc 6 months a year. During the next 4 years no recurrence has been observed

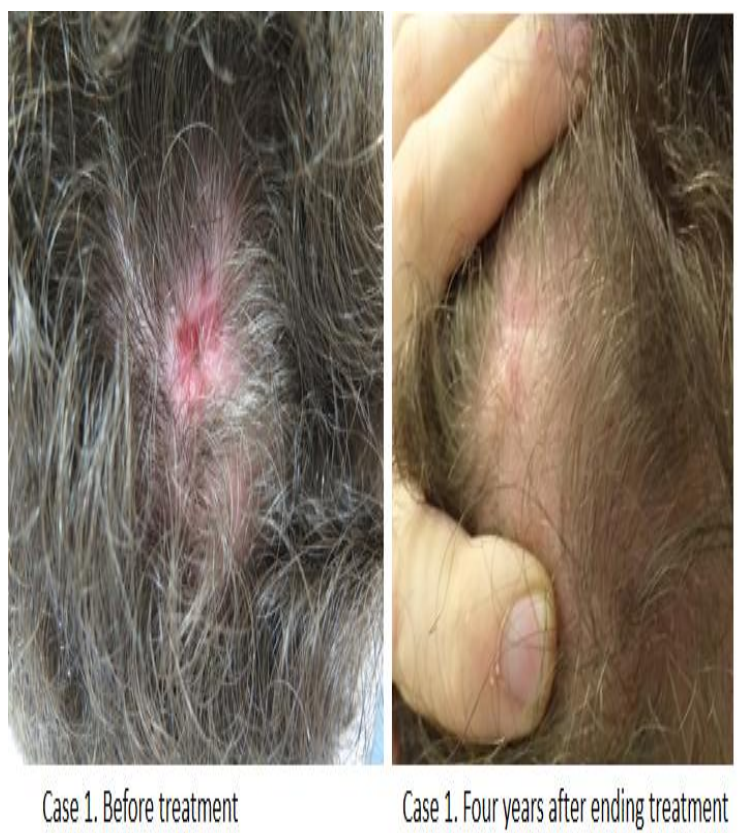

Fig1: Case 1. Before treatment. Four years after ending treatment.

\subsection{Case 2}

A 16-year-old man reported having had itching, crusting and erythema of the scalp for 3 years resistant to oral and topical therapies. He had a family history of hidrosadenitis and his sister with nor affiliated inflammatory lesions on the scalp. In the examination a $2 \times 3 \mathrm{~cm}$ scarring alopecia patch on the vertex was observed. Trichoscopic findings include tufting, perifollicular erythema, crusts and follicular keratosis . A skin biopsy showed unspecific inflammatory changes. Laboratory results elevated levels of uric acid. The remaining parameters were within the normal range. Following the diagnosis of scarring primary alopecia type folliculitis decalvans. Nearly complete healing was seen after the same treatment regimen that case 1.(Fig 2) No relapse has occurred during a four years of follow-up.
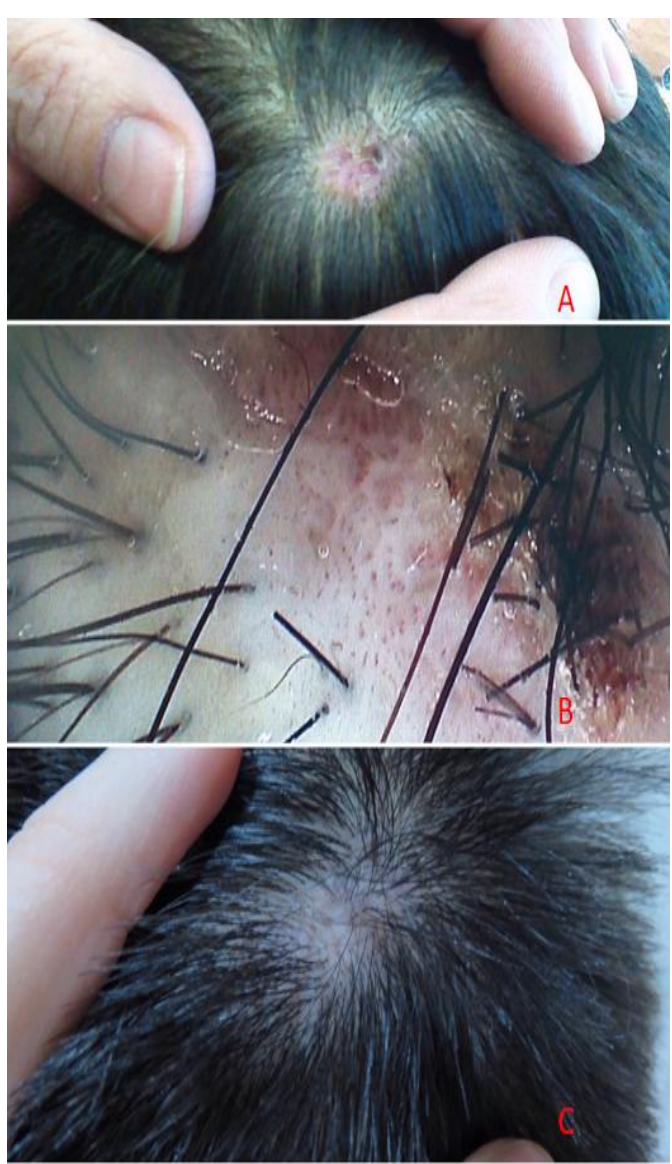

Case 2. A Before treatment B. Trichoscopy C. Six months after treatment

Fig2: Case 2. A .Before treatment B. Trichoscopy $C$. Six months after treatment
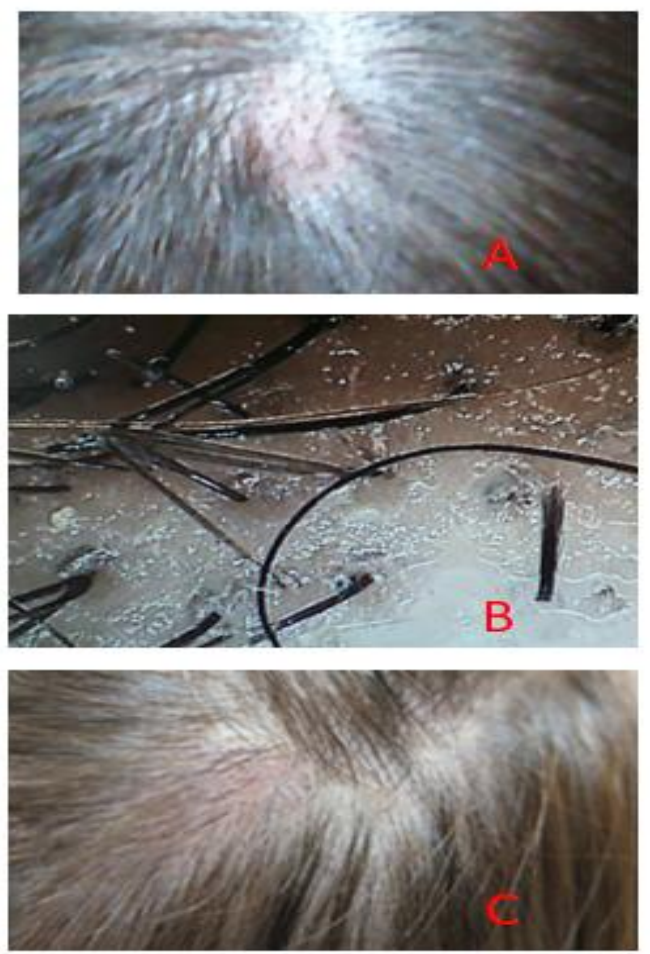

Case 3 . A Before treatment B Trichoscopy $C$ Six months after treatment

Fig3: Case 3. A Before treatment B Trichoscopy $C$. Six months after treatment. 


\subsection{Case 3}

A 22-year-old women, case 2 sister, was admitted with a $0,5 \times 1 \mathrm{~cm}$ area of scarring alopecia at the vertex. Trichoscopy showed, "tufted" folliculitis and perifollicular casts consistent with folliculitis decalvans. She was treated with laser Nd YAG (a total of 2 sessions in a 4 week interval ) and alopecia improved without relapse after three years of follow up. (Fig 3)

\subsection{Case 4}

A 38-year-old male with personal history of hidradenitis, smoking, dislipemia and acne treated with isotretinoin, presented to us with a scarring alopecia in his face. On examination an alopecic and supurative plaque on his left cheek was observed. Histopathological study demonstrates perifolliculits. Dissecting cellulitis was suspected. The patient improved after treatment with gluconate zinc oral and lasertherapy (4 sessions with laser Nd-YAG). (Fig 4)No relapse has occurred during three years of follow-up.

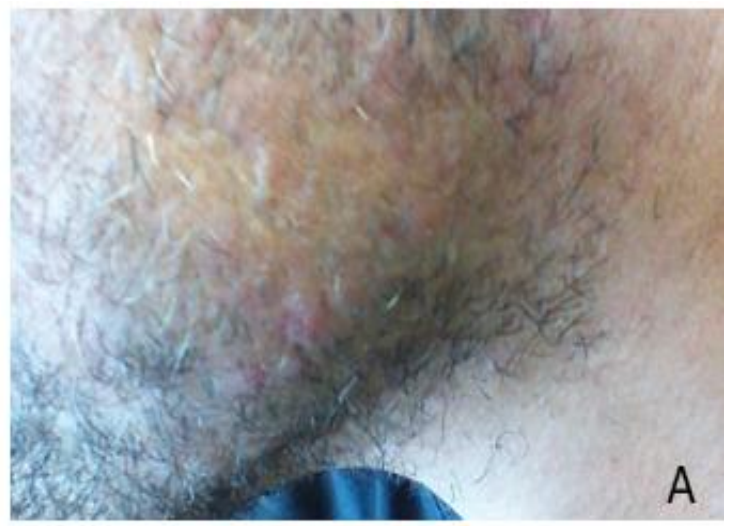

A Before treatment (August 2016)

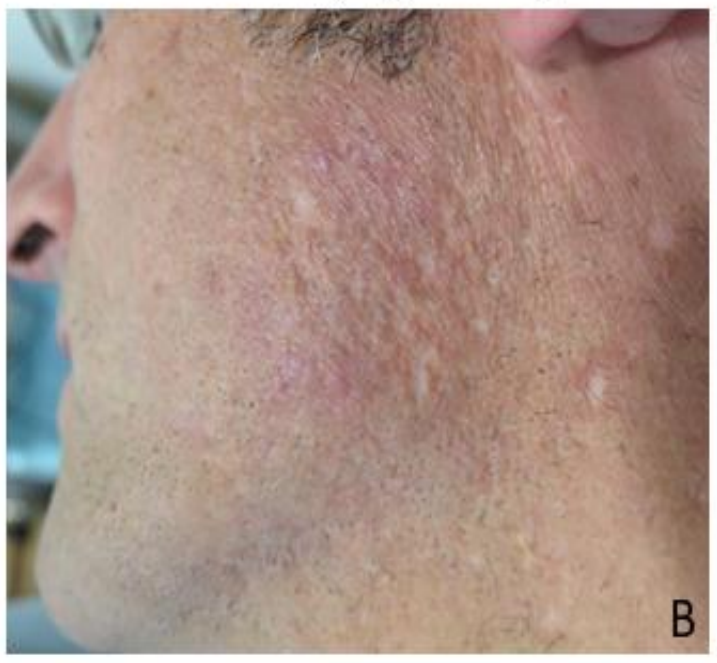

B October 2017 (last sesion May 2017)

Fig4: Case 4. A Before treatment (August 2016). B October 2017 (last sesion May 2017)

\subsection{Case 5}

A 29-year-old male was referred to our department because a patch of localized alopecia in his occipital region. A histopatholohigal study showed histological features consistent with folliculitis decalvans. $\mathrm{He}$ has been successfully treated with oral gluconate zinc $70 \mathrm{mg}$ daily (6 months a year) combined with Nd:YAG laser (the same treatment regimen that case 1) without relapse after 7 months of follow up. (Fig 5)

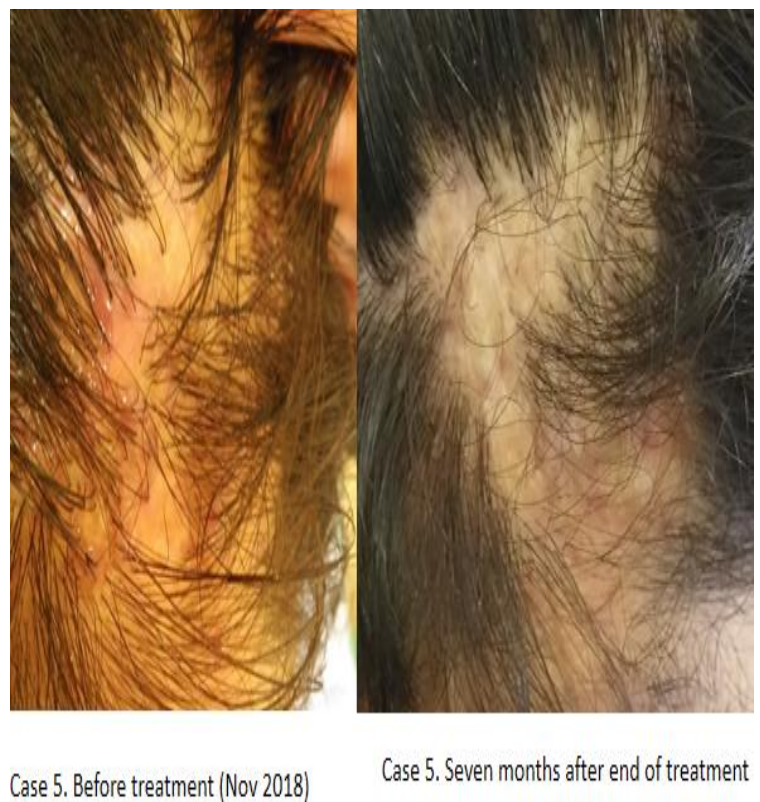

Fig5: Case 5. Before treatment (Nov 2018). Seven months after end of treatment

\section{DISCUSSION}

PCA includes conditions of varied clinical and pathological features. The newer insights into the pathogenesis of PAC mainly involve the HFSCs (hair follicle slow-cycling pluripotent cells located in the "bulge" region of hair follicles in outer root sheath destruction theories, impairment of self-maintenance, lipid metabolism alteration, and neurogenic inflammation theory, environmental and genetic factors [1])

A working classification of PCA, mainly based on the most representative pathological finding of scalp biopsy sample include chronic cutaneous lupus erythematosus (CCLE), lichen planopilaris (LPP), classic pseudopelade of Brocq (PPB), central centrifugal cicatricial alopecia (CCCCA), alopecia mucinosa (AM) and keratosis follicularis spinulosa decalvans (KFSD) as "lymphocytic" PCA. Frontal fibrosing alopecia (FFA) and Graham-Little syndrome (GLS) were considered as LLP 
variants. The neutrophilic PCA group comprises FD and dissecting cellulitis/folliculitis (DC/DF). Acne keloidalis (AK), acne necrotica (AN) and eruptive pustular dermatosis (EPD) were classified as mixed cell infiltrate PCA. Nonspecific cicatricial alopecia was defined as "idiopathic scarring" with inconclusive clinical and histopathological findings [6].

$\mathrm{CA}$ are a diagnostic and therapeutic challenge. An accurate clinical assessment, based on a careful patient history, integrated with microbiological studies, dermoscopy and histopathologic studies permit and early diagnosis. Trichoscopy has become a standard method in differential diagnosis of hair and scalp disorders.

On the basis of histology, a differentiation is made between primary cicatricial alopecias (lymphocyte-mediated, neutrophil-mediated and mixed) due to preferential destruction of the follicle, and secondary cicatricial alopecias when the follicle secondarily damages as result of a more generalized destructive process as trauma (burns, radiation, traction), infiltrative processes (morphea, scleroderma, sarcoidosis, neoplasias) and infections (bacterial, fungal, viral, mycobacterial). Direct immunofluorescence study is important to differentiate between lichen planus pilaris or cutaneous lupus erythematosus. Microarray analysis of global gene expression profile may mark a new era in the diagnosis of PCA. Recently a report of microarray analysis generated from total RNA in lesions of LPP y PPB suggested that the two conditions are biologically distinct $[1,5]$

The aim of treatment currently focuses the reduction of symptoms and to slow or stop the progression of the inflammatory waves and the scarring process at the earliest phase of involvement. Given the role of cytotoxic autoimmune response and host predisposition to S. aureus infection of patients with PCA the first-line medications generally selected are immunosuppressive agents for lymphocytic PCA and antimicrobials or dapsone for the neutrophilic PCA. New insights into the etiopathogenesis of PCA imply the use of more efficient drugs PPAR- $\square$ agonist may provide promising remedies for LPP. CD200 agonists might be beneficial in PCA treatment. [5]

DC or Hoffmann disease is a rare primary neutrophilic cicatricial alopecia, characterized by a profound, chronic and recurrent folliculitis, ending in abscesses and sinuous tracts and scars that can occur with acne conglobate, hidradenitis suppurativa and pilonidal cysts within "the follicular occlusion triad" and most of the times resistant to the administered therapies. Different therapeutic approaches, such as monotherapy by isotretinoin, acitretin, isotretinoin combined with corticosteroids, adalimumab, antibiotics, zinc and hormonal treatments have been used. Ablation of the follicle could also play a role in the treatment of DC and laser (800 nm diode laser, 694nm longpulse non Q-switched ruby laser, long-pulsed Nd: YAG) or X-ray therapy have been used successfully [7,]. Some authors reported healing after zinc substitution therapy. $[7,8]$

Folliculitis decalvans (FD) of Quinquaud is a neutrophilic CA. that occurs predominantly in middle-age males. The therapeutic approach, usually focused on the eradication of S. aureus anti-inflammatory agents, is problematic. A complete remission after treatment with the long-pulsed neodymium: yttrium aluminium garnet (Nd:YAG) laser has been reported [9,10]. Recently Miguel-Lopez at al [11] reported good results after photodynamic therapy in 10 patients. Depending upon the duration of stable disease, the surgical therapies are recommended after at least $1-2$ years of quiescence [1].

Zinc was shown to be a cofactor for metalloenzimes and transcription factor involved in many cellular processes. The antioxidant and anti-inflammatory properties of zinc have been postulated to be useful in the management different skin disorders $[12,13]$ as well as in the treatment of the follicular occlusion disorders like folliculitis decalvans and perifolliculitis capitis abscedens et suffodiens .[14- 15 ] Laser epilation has been used with success in the management of different primary cicatricial alopecias. [16-18] According to some authors, removal of residual follicles resulting in permanent elimination of the inflammatory focus could be the reason why laser therapy would improve scarring inflammatory alopecias and Nd: YAG 1064-nm laser penetrates deeper, therefore, is the best for treating very dark patients. $[9,10]$

Scarring alopecia is a common reason for consultation. Su et al [19] reviewed 89 patients with pathology-confirmed PCA. The most common type was dissecting cellulitis (DC) $(30.3 \%)$, followed by lichen planopilaris (LPP) 
$(23.5 \%)$, central centrifugal cicatricial alopecia (CCCA) (12.4\%) and acne keloidalis nuchae (AKN) (12.4\%). Neutrophilic PCA are more common in young males and management of these patients is difficult. In our experience laser therapy has been effective and safe therapy

\section{Conclusion}

In conclusion, our results show the utility of a combination of oral zinc and laser therapy and may be an option for the treatment of some cases of PCA. Long-term studies with a larger number of patients are needed.

\section{REFERENCES}

[1] Dogra S, Sarangal R. What's new in cicatricial alopecia? Indian J Dermatol Venereol Leprol 2013; 79: 576-90

[2] Rongioletti F, Christiana K. Cicatricial (scarring) alopecias: an overview of pathogenesis, classification, diagnosis, and treatment. Am J Dermatol 2012; 13: 247-60

[3] Abal-Diaz L, Soria X, Casanova-Seuma JM. Alopecias cicatriciales. Actas Dermosifiliogr 2012; 103:376-387

[4] Ross EK, Tan E, Shapiro J. Update on primary cicatricial alopecias. J Am Acad Dermatol 2005; 53: 1-37

[5] Ohyama M. Primary cicatricial alopecia: Recent advances in understanding and management J of Dermatol 2012:39:18-26

[6] Olsen EA, Bergfeld WF, Cotsarelis G, Price VH, Shapiro J, Sinclair R et al. Summary of North American Hair Research Society (NAHRS)- sponsored workshop on cicatricial alopecia, Duke University Medical Center , February 1011, 2001. J Am Acad Dermatol 2003;48:103-10

[7] Scheinfeld N. Dissecting cellulitis (perifolliculitis Capitis Abscedens et suffodiens): a comprehensive review focusing on new treatments and findings of the last decade with commentary comparing the therapies and causes of dissecting cellulitis to hidradenitis suppurativa. Dermatology Online J 2014; 20 (5)
[8] Jacobs F, Metzler G, Kubiak J, Röcken M, Schaller M. New approach in combined therapy of perofolliculitis capitis Abscedens et suffodiens. Acta Dermato Venereol 2011; 91:726-7

[9] Meesters AA, Van der Veen JP, Wolkerstorfer A. Long-term remission of folliculitis decalvans after treatment with the long-pulsed Nd:YAG laser. J of Dermatol Treat 2014;25: 167-168

[10] Parlette EC, Kroeger N, Ross EV. Nd:YAG laser treatment of recalcitrant folliculitis decalvans. Dermatol Surg. 2004; 30:1152-4.

[11] Miguel-Gomez L, Vano-Galvan S, PerezGarcia B, Carrillo-Gijon R, Jaen-Olasolo P. Treatment of folliculitis decalvans with photodynamic therapy: results in 10 patients. $\mathrm{J}$ Am Acad Dermatol 2015;72:1085-7

[12] Gupta M, Mahajan VK, Mehta KS, Chauhan PS. Zinc therapy in dermatology: a review. Dermatol Res Pract. 2014;2014:709152

[13] Bae YS, Hill ND, Bibi Y, Dreiher J, Cohen AD. Innovative uses for zinc in dermatology. Dermatol Clin. 2010 ;28:587-97

[14] Berne B, Venge P, Ohman S. Perifolliculitis capitis abscedens et suffodiens (Hoffman). Complete healing associated with oral zinc therapy. Arch Dermatol. 1985 121:1028-30.

[15] Abeck D, Korting HC, Braun-Falco O. Folliculitis decalvans. Long-lasting response to combined therapy with fusidic acid and zinc. Acta Derm Venereol. 1992;72:143-5.

[16] Otberg N. Primary cicatricial alopecias. Dermatol Clin. 2013 ;31:155-66.

[17] Bolduc C, Sperling LC, Shapiro J. Primary cicatricial alopecia: Other lymphocytic primary cicatricial alopecias and neutrophilic and mixed primary cicatricial alopecias. J Am Acad Dermatol. 2016 ;75:1101-1117

[18] Kanti V, Röwert-Huber J, Vogt A, BlumePeytavi U. Cicatricial alopecia. J Dtsch Dermatol Ges. $2018 ; 16: 435-461$

[19] Su HJ, Cheng AY, Liu CH, Chu CB, Lee CN, Hsu CK, Lee JY, Yang CC. Primary scarring alopecia: A retrospective study of 89 patients in Taiwan. J Dermatol. 2018;45:450-455.

Citation: Rosa Giménez-García, Dra Teresa E. Lázaro Cantalejo, Primary Cicatricial Alopecia Successfully Treated with A Combination of Oral Zinc with Nd: YAG Laser. A Case Series of Five Patients. ARC Journal of Dermatology. 2020; 5(1):7-11. doi:dx.doi.org/10.20431/2456-0022.0501002.

Copyright: () 2020 Authors. This is an open-access article distributed under the terms of the Creative Commons Attribution License, which permits unrestricted use, distribution, and reproduction in any medium, provided the original author and source are credited. 